Bull. Korean Math. Soc. 47 (2010), No. 6, pp. 1139-1153

DOI 10.4134/BKMS.2010.47.6.1139

\title{
SELF-NORMALIZED WEAK LIMIT THEOREMS FOR A $\phi$-MIXING SEQUENCE
}

\author{
Yong-Kab Choi And HeE-Jin Moon
}

\begin{abstract}
Let $\left\{X_{j}, j \geq 1\right\}$ be a strictly stationary $\phi$-mixing sequence of non-degenerate random variables with $\mathrm{E} X_{1}=0$. In this paper, we establish a self-normalized weak invariance principle and a central limit theorem for the sequence $\left\{X_{j}\right\}$ under the condition that $L(x):=\mathrm{E} X_{1}^{2} I\left\{\left|X_{1}\right| \leq\right.$ $x\}$ is a slowly varying function at $\infty$, without any higher moment conditions.
\end{abstract}

\section{Introduction and results}

Csörgö et al. [6] proved the following self-normalized weak invariance principle for a sequence of i.i.d. centered random variables: Let $\left\{X_{j}, j \geq 1\right\}$ be a sequence of non-degenerate i.i.d. random variables with zero means on the probability space $(\Omega, \mathcal{F}, \mathrm{P})$, and let $\mathbf{S}_{n}=\sum_{j=1}^{n} X_{j}, V_{n}^{2}=\sum_{i=1}^{n} X_{i}^{2}$. Then, on the appropriate probability space, one can construct a standard Wiener process $\{W(t), t \geq 0\}$ such that

$$
\sup _{0 \leq t \leq 1}\left|\frac{\mathbf{S}_{[n t]}}{V_{n}}-\frac{W(n t)}{\sqrt{n}}\right| \stackrel{\mathrm{P}}{\rightarrow} 0, \quad n \rightarrow \infty
$$

if and only if

$$
L(x):=\mathrm{E} X_{1}^{2} I\left\{\left|X_{1}\right| \leq x\right\} \text { is a slowly varying function at } \infty .
$$

Other related results for self-normalized limit theory have been developed by many authors, e.g., the LIL was obtained in Griffin and Kuelbs [8], the large deviation principle can be found in Shao [15], the lag increment theorems in Wang [16] and Csörgö et al. [5], and the functional central limit theorem in Račkauskas and Suquet [12].

On the other hand, consider a sequence of dependent random variables $\left\{X_{j} ; j \geq 1\right\}$. Let $\left\{X_{j} ; j \geq 1\right\}$ be a strictly stationary sequence of random variables on $(\Omega, \mathcal{F}, \mathrm{P})$. Set $\mathcal{F}_{a}^{b}=\sigma\left(X_{i} ; a \leq i \leq b\right)$, a $\sigma$-algebra generated by

Received December 26, 2008; Revised July 6, 2010.

2000 Mathematics Subject Classification. Primary 60F15, 60F17.

Key words and phrases. self-normalized random variables, invariance principle, central limit theorem, mixing sequence.

Research supported by NRF(03-2009-0075). 
$X_{i}$ for $a \leq i \leq b$, where $1 \leq a \leq b<\infty$. Then we say that $\left\{X_{j}, j \geq 1\right\}$ is a $\phi$-mixing sequence if $\phi(n) \rightarrow 0$ as $n \rightarrow \infty$, where

$$
\phi(n)=\sup _{m \geq 1} \sup _{\substack{A \in \mathcal{F}_{1}^{m},,_{B \in \mathcal{F}_{m+n}^{\infty}}(A) \neq 0 \\ B \in \mathcal{F}_{m+n}}}|\mathrm{P}(B \mid A)-\mathrm{P}(B)|,
$$

while $\left\{X_{j}, j \geq 1\right\}$ is said to be a $\rho$-mixing sequence if $\rho(n) \rightarrow 0$ as $n \rightarrow \infty$, where

$$
\rho(n)=\sup _{m \geq 1} \sup _{\substack{f \in L_{2}\left(\mathcal{F}_{1}^{m}\right) \\ g \in L_{2}\left(\mathcal{F}_{m+n}^{\infty}\right)}}|\operatorname{corr}(f, g)| .
$$

It is well-known that $\rho(n) \leq 2 \phi^{1 / 2}(n)$. Hence a $\phi$-mixing sequence is $\rho$-mixing. In the sequel, the following notations will be used: $S_{j}(k)=\sum_{i=j+1}^{j+k} X_{i}$ for $j \geq$ $0, S_{n}=\sum_{i=1}^{n} X_{i},[x]$ denotes the integer part of $x, I(\cdot)$ is the indicator function and " $\Rightarrow$ " denotes the weak convergence in the space $D[0,1]$ with Skorohod topology.

Recently, Balan and Kulik [1] obtained the following self-normalized weak invariance principle for a strictly stationary $\phi$-mixing sequence of random variables, which may be motivated by the central limit theorem of Bradley [4] and the invariance principles of Shao ([13], [14]): Let $\left\{X_{j}, j \geq 1\right\}$ be a strictly stationary $\phi$-mixing sequence of non-degenerate random variables such that $\mathrm{E} X_{1}=0$ and (1.2) holds. Suppose that $\phi(1)<1$ and $\sum_{n=1}^{\infty} \phi^{1 / 2}(n)<\infty$. Then, on an appropriate probability space,

$$
\sup _{0 \leq t \leq 1}\left|\frac{S_{[n t]}}{\beta V_{n}}-\frac{W\left(s_{[n t]}^{2}\right)}{s_{n}}\right| \stackrel{\mathrm{P}}{\rightarrow} 0, \quad n \rightarrow \infty
$$

for some suitable constants $s_{k}^{2}$ and positive constant $\beta$.

For our purpose, let us introduce the following conditions and notations. Let $\left\{X_{j}, j \geq 1\right\}$ be a strictly stationary $\phi$-mixing sequence of non-degenerate random variables with $\mathrm{E} X_{1}=0$, and let $\left\{\ell_{n}, n \geq 1\right\}$ be a sequence of positive integers such that $1 \leq \ell_{n} \leq n, \ell_{n} \rightarrow \infty, \ell_{n}=o(n)$, as $n \rightarrow \infty$, and further $\ell_{n}$ is slowly varying. Write $\ell=\ell_{n}$ and set, for each $\ell$,

$$
B_{n}^{2}=\frac{1}{n-\ell+1} \sum_{i=0}^{n-\ell}\left(\frac{S_{i}(\ell)}{\sqrt{\ell}}\right)^{2} .
$$

In order to make the central limit theorem applicable in practice from the given data, Peligrad and Shao [11] used a self-normalizer $\sqrt{\ell} B_{n}$ for $S_{n}$ and proved the central limit theorem

$$
\frac{S_{n}}{\sqrt{\ell} B_{n}} \stackrel{\mathcal{D}}{\rightarrow} N(0,1), \quad n \rightarrow \infty
$$

under the centered stationary $\rho$-mixing sequence assumption with $\mathrm{E}\left(X_{1}\right)^{2}<\infty$. 
Let $\left\{X_{j}, j \geq 1\right\}$ be a strictly stationary $\phi$-mixing sequence of non-degenerate random variables such that $\mathrm{E} X_{1}=0$, and let

$$
L(x):=\mathrm{E} X_{1}^{2} I\left\{\left|X_{1}\right| \leq x\right\} \text { is a slowly varying function at } \infty .
$$

The aim of this paper is to obtain a self-normalized weak invariance principle and a central limit theorem for the strictly stationary $\phi$-mixing sequence of the forms (1.1) and (1.3) by using the self-normalizer $\sqrt{\ell} B_{n}$ instead of $\beta V_{n}$, under the condition (1.4) without any higher moment conditions.

Set $b=\inf \{x \geq 1: L(x)>0\}$ and define

$$
z_{n}=\inf \left\{s: s \geq b+1, \frac{L(s)}{s^{2}} \leq \frac{1}{n \ell}\right\}, \quad n \geq 1 .
$$

One can easily obtain the following properties on $z_{n}$ and $L(\cdot)$ :

$$
n \ell L\left(z_{n}\right)=z_{n}^{2} \text { and } z_{n} \rightarrow \infty \text { as } n \rightarrow \infty \text {. }
$$

We refer the reader to [2] and [4] for more details of these $z_{n}$ and $L(\cdot)$.

For convenience, we denote that, for each $j=1,2, \ldots$,

$$
Y_{j, n}=X_{j} I\left(\left|X_{j}\right| \leq z_{n}\right), \quad \bar{Y}_{j, n}=X_{j}-Y_{j, n}=X_{j} I\left(\left|X_{j}\right|>z_{n}\right), \quad n \geq 1,
$$

and set for each $k=1,2, \ldots$ and $i \geq 0$

$$
\begin{gathered}
S_{i}^{(n)}(k)=\sum_{j=i+1}^{i+k} Y_{j, n}, \quad \bar{S}_{i}^{(n)}(k)=\sum_{j=i+1}^{i+k} \bar{Y}_{j, n}, \\
T_{i}^{(n)}(k)=\sum_{j=i+1}^{i+k}\left(Y_{j, n}-\mathrm{E} Y_{j, n}\right), \quad \bar{T}_{i}^{(n)}(k)=\sum_{j=i+1}^{i+k}\left(\bar{Y}_{j, n}-\mathrm{E} \bar{Y}_{j, n}\right), \\
S_{k}^{(n)}=\sum_{j=1}^{k} Y_{j, n}, \quad T_{k}^{(n)}=\sum_{j=1}^{k}\left(Y_{j, n}-\mathrm{E} Y_{j, n}\right) .
\end{gathered}
$$

Clearly, $S_{i}(k)=S_{i}^{(n)}(k)+\bar{S}_{i}^{(n)}(k)=T_{i}^{(n)}(k)+\bar{T}_{i}^{(n)}(k)$. Finally, we define for each $k, n=1,2, \ldots$

$$
\nu_{k}=\nu_{k}^{(n)}=\left\{\operatorname{Var}\left(S_{k}^{(n)}\right)\right\}^{1 / 2} \text { and } \gamma_{k}=\gamma_{k}^{(n)}=\nu_{k} /\left\{\ell L\left(z_{n}\right)\right\}^{1 / 2} .
$$

Our main results are as follows:

Theorem 1.1. Let $\left\{X_{j}, j \geq 1\right\}$ be a strictly stationary $\phi$-mixing sequence of non-degenerate random variables such that $\mathrm{E} X_{1}=0$ and the condition (1.4) holds. Suppose that $\phi(1)<1$ and $\sum_{n=1}^{\infty} \phi^{1 / 2}(n)<\infty$. Then, on an appropriate probability space for $X_{1}, X_{2}, \ldots$, we can construct a standard Wiener process $\{W(t), 0 \leq t<\infty\}$ such that

$$
\sup _{0 \leq t \leq 1}\left|\frac{S_{[\ell t]}}{\sqrt{\ell} B_{n}}-\frac{W\left(s_{[\ell t]}^{2}\right)}{s_{\ell}}\right| \stackrel{\mathrm{P}}{\rightarrow} 0, \quad n \rightarrow \infty
$$


for some suitable constants $s_{k}^{2}$, which will be specified later on (see the proof of Proposition 2.1 below).

Theorem 1.2. Under the assumptions of Theorem 1.1, we have, as $n \rightarrow \infty$,

$$
\frac{S_{\ell}}{\sqrt{\ell} B_{n}} \stackrel{\mathcal{D}}{\rightarrow} N(0,1) \quad \text { and } \quad \frac{S_{[\ell t]}}{\sqrt{\ell} B_{n}} \Rightarrow W(t) \quad \text { for } 0 \leq t \leq 1
$$

\section{Proofs}

The proofs of Theorems 1.1 and 1.2 will be accomplished through the following several lemmas and Propositions 2.1-2.4.

Lemma $2.1([11])$. Let $\left\{X_{j}, j \geq 1\right\}$ be a $\rho$-mixing sequence of random variables with $\mathrm{E} X_{j}=0$ and $\mathrm{E} X_{j}^{2}<\infty$. Then

$$
\mathrm{E}\left(\sum_{i=1}^{n} X_{i}\right)^{2} \leq C \cdot \exp \left(2 \sum_{i=0}^{\left[\log _{2} n\right]} \rho\left(2^{i}\right)\right) \cdot n \cdot \max _{i \leq n} \mathrm{E} X_{i}^{2} .
$$

Lemma 2.2 ([11]). Suppose that $\left\{X_{j}, j \geq 1\right\}$ is a $\rho$-mixing sequence of random variables. Let $\left\{\ell_{n}, n \geq 1\right\}$ be a sequence of integers with $1 \leq \ell_{n} \leq$ $n$, and let $f$ be a real-valued Borel measurable function on $\mathbb{R}^{\ell_{n}}$. Put $Z_{j}=$ $f\left(X_{j+1}, \ldots, X_{j+\ell_{n}}\right)$. Then we have

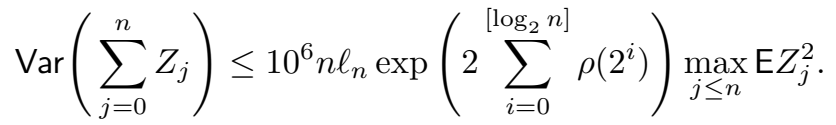

Lemma 2.3 ([10]). Let $\left\{X_{j}, j \geq 1\right\}$ be a strictly stationary sequence with $\mathrm{E} X_{1}=0$ and $\sigma_{n}^{2}:=\operatorname{Var}\left(S_{n}\right) \rightarrow \infty$. Suppose that a function $q:[0, \infty) \rightarrow[0, \infty)$ satisfies the conditions: $(\mathrm{A} 1) q$ is continuous and $q(0)=0 ;(\mathrm{A} 2) q(x) / x^{2+\delta_{0}}$ is nondecreasing for some $\delta_{0}>0$ (for all $x$ sufficiently large); (A3) $q(2 x) \leq c q(x)$ with some constant $c>0$ (for all $x$ sufficiently large). Then there is a constant $K>0$ such that

$$
\mathrm{E} q\left(\max _{1 \leq i \leq n}\left|S_{i}\right| / \sigma_{n}\right) \leq K \quad \text { for every } n \geq 1 .
$$

Lemma 2.4. For any real vectors $\left(x_{1}, \ldots, x_{n}\right),\left(y_{1}, \ldots, y_{n}\right) \in \mathbb{R}^{n}, n=1,2, \ldots$, and any positive real $c$, we have

$$
\left.|| \sum_{i=1}^{n}\left(x_{i}+y_{i}\right)^{2}\right|^{1 / 2}-c^{1 / 2}|\leq| \sum_{i=1}^{n} x_{i}^{2}-\left.c\right|^{1 / 2}+\left|\sum_{i=1}^{n} y_{i}^{2}\right|^{1 / 2} .
$$

Proof. By the Minkowski inequality and the elementary inequality $\sqrt{a}-\sqrt{b} \leq$ $\sqrt{|a-b|}$ for $a, b \geq 0$, we have

$$
\left|\sum_{i=1}^{n}\left(x_{i}+y_{i}\right)^{2}\right|^{1 / 2}-c^{1 / 2} \leq\left|\sum_{i=1}^{n} x_{i}^{2}\right|^{1 / 2}+\left|\sum_{i=1}^{n} y_{i}^{2}\right|^{1 / 2}-c^{1 / 2}
$$


SELF-NORMALIZED WEAK LIMIT THEOREMS FOR A $\phi$-MIXING SEQUENCE 1143

$$
\leq\left|\sum_{i=1}^{n} x_{i}^{2}-c\right|^{1 / 2}+\left|\sum_{i=1}^{n} y_{i}^{2}\right|^{1 / 2} .
$$

On the other hand, using the inequality $\|x+y\|_{2} \geq\|x\|_{2}-\|y\|_{2}$ yields

$$
\begin{aligned}
c^{1 / 2}-\left|\sum_{i=1}^{n}\left(x_{i}+y_{i}\right)^{2}\right|^{1 / 2} & \leq c^{1 / 2}-\left|\sum_{i=1}^{n} x_{i}^{2}\right|^{1 / 2}+\left|\sum_{i=1}^{n} y_{i}^{2}\right|^{1 / 2} \\
& \leq\left|c-\sum_{i=1}^{n} x_{i}^{2}\right|^{1 / 2}+\left|\sum_{i=1}^{n} y_{i}^{2}\right|^{1 / 2} .
\end{aligned}
$$

Lemma 2.5. Let $\left\{X_{j}, j \geq 1\right\}$ be a strictly stationary sequence of nondegenerate random variables with $\mathrm{E} X_{1}=0$. Suppose that $\sum_{n=1}^{\infty} \rho\left(2^{n}\right)<\infty$. Then, for the $\gamma_{\ell}$ in (1.5), we have

$$
\frac{B_{n}}{\sqrt{L\left(z_{n}\right)}}-\gamma_{\ell} \rightarrow 0 \quad \text { as } n \rightarrow \infty \quad \text { in the mean. }
$$

Proof. From Lemma 2.4, it follows that

$$
\begin{aligned}
&\left|\frac{B_{n}}{\sqrt{L\left(z_{n}\right)}}-\gamma_{\ell}\right| \\
&= \frac{1}{\sqrt{L\left(z_{n}\right)}}\left|B_{n}-\left\{\operatorname{Var}\left(S_{\ell}^{(n)}\right) / \ell\right\}^{1 / 2}\right| \\
&= \frac{1}{\sqrt{\ell(n-\ell+1) L\left(z_{n}\right)}}\left|\left(\sum_{i=0}^{n-\ell}\left(S_{i}(\ell)\right)^{2}\right)^{1 / 2}-\left(\sum_{i=0}^{n-\ell} \operatorname{Var}\left(S_{\ell}^{(n)}\right)\right)^{1 / 2}\right| \\
&= \frac{1}{\sqrt{\ell(n-\ell+1) L\left(z_{n}\right)}} \mid\left(\sum_{i=0}^{n-\ell}\left(T_{i}^{(n)}(\ell)+\bar{T}_{i}^{(n)}(\ell)\right)^{2}\right)^{1 / 2} \\
& \leq \frac{1}{\sqrt{\ell(n-\ell+1) L\left(z_{n}\right)}}\left|\sum_{i=0}^{n-\ell}\left\{T_{i}^{(n)}(\ell)\right\}^{2}-\mathrm{E}\left(\sum_{i=0}^{n-\ell}\left\{T_{i}^{(n)}(\ell)\right\}^{2}\right)\right|^{1 / 2} \\
& \quad+\frac{1}{\sqrt{\ell(n-\ell+1) L\left(z_{n}\right)}}\left(\sum_{i=0}^{n-\ell}\left\{\bar{T}_{i}^{(n)}(\ell)\right\}^{2}\right)^{1 / 2}
\end{aligned}
$$


We first compute $I_{1}$. By the Hölder inequality, we have

$$
\begin{aligned}
& \mathrm{E}\left|\sum_{i=0}^{n-\ell}\left\{T_{i}^{(n)}(\ell)\right\}^{2}-\mathrm{E}\left(\sum_{i=0}^{n-\ell}\left\{T_{i}^{(n)}(\ell)\right\}^{2}\right)\right|^{1 / 2} \\
\leq & \left\{\mathrm{E}\left(\sum_{i=0}^{n-\ell}\left\{T_{i}^{(n)}(\ell)\right\}^{2}-\mathrm{E}\left(\sum_{i=0}^{n-\ell}\left\{T_{i}^{(n)}(\ell)\right\}^{2}\right)\right)^{2}\right\}^{1 / 4} \\
= & \left\{\operatorname{Var}\left(\sum_{i=0}^{n-\ell}\left\{T_{i}^{(n)}(\ell)\right\}^{2}\right)\right\}^{1 / 4} .
\end{aligned}
$$

By Lemma 2.2 with $Z_{i}:=\left\{T_{i}^{(n)}(\ell)\right\}^{2}$, we have

$\operatorname{Var}\left(\sum_{i=0}^{n-\ell}\left\{T_{i}^{(n)}(\ell)\right\}^{2}\right) \leq C \cdot(n-\ell) \cdot \ell \cdot \exp \left(2 \sum_{i=0}^{\left[\log _{2}(n-\ell)\right]} \rho\left(2^{i}\right)\right) \max _{0 \leq i \leq n-\ell} \mathrm{E}\left\{T_{i}^{(n)}(\ell)\right\}^{4}$. Applying Lemma 2.3 to the sequence $\left\{Y_{j, n}-\mathrm{E} Y_{j, n} ; 1 \leq j \leq \ell\right\}$ and the function $q(x)=x^{4}$, we have

$$
\mathrm{E}\left\{T_{i}^{(n)}(\ell)\right\}^{4}=\mathrm{E}\left\{T_{\ell}^{(n)}\right\}^{4} \leq K\left(\operatorname{Var} T_{\ell}^{(n)}\right)^{2}=K\left(\mathrm{E}\left\{T_{\ell}^{(n)}\right\}^{2}\right)^{2} .
$$

It is easily seen from Lemma 2.1 that

$$
\begin{aligned}
\mathrm{E}\left\{T_{\ell}^{(n)}\right\}^{2} & =\mathrm{E}\left(\sum_{j=1}^{\ell}\left(Y_{j, n}-\mathrm{E} Y_{j, n}\right)\right)^{2} \\
& \leq C \ell \exp \left(2 \sum_{i=0}^{\left[\log _{2}(n-\ell)\right]} \rho\left(2^{i}\right)\right) \max _{1 \leq i \leq \ell} \mathrm{E}\left\{Y_{j, n}-\mathrm{E} Y_{j, n}\right\}^{2} \\
& \leq C \ell L\left(z_{n}\right) .
\end{aligned}
$$

Combining these results, we obtain

$$
\begin{aligned}
\mathrm{E} I_{1} & \leq C \frac{\left\{\ell^{3}(n-\ell)\left(L\left(z_{n}\right)\right)^{2}\right\}^{1 / 4}}{\sqrt{\ell(n-\ell+1) L\left(z_{n}\right)}}=C\left(\frac{\ell^{3}(n-\ell)\left(L\left(z_{n}\right)\right)^{2}}{\ell^{2}(n-\ell+1)^{2}\left(L\left(z_{n}\right)\right)^{2}}\right)^{1 / 4} \\
& =C\left(\frac{\ell}{n-\ell+1}\right)^{1 / 4}=o(1), \quad n \rightarrow \infty
\end{aligned}
$$

We next compute $I_{2}$. It is immediate that

$$
\begin{aligned}
\mathrm{E}\left(\sum_{i=0}^{n-\ell}\left\{\bar{T}_{i}^{(n)}(\ell)\right\}^{2}\right)^{1 / 2} & \leq \mathrm{E}\left(\sum_{i=0}^{n-\ell}\left|\bar{T}_{i}^{(n)}(\ell)\right|\right)=\sum_{i=0}^{n-\ell} \mathrm{E}\left|\bar{T}_{i}^{(n)}(\ell)\right| \\
& \leq 2 \sum_{i=0}^{n-\ell} \sum_{j=i+1}^{i+\ell} \mathrm{E}\left|X_{j}\right| I\left(\left|X_{j}\right|>z_{n}\right) \\
& =2 \ell(n-\ell+1) \mathrm{E}\left|X_{1}\right| I\left(\left|X_{1}\right|>z_{n}\right) .
\end{aligned}
$$


SELF-NORMALIZED WEAK LIMIT THEOREMS FOR A $\phi$-MIXING SEQUENCE 1145

By the argument in the proof of Lemma 3.1 in [4], we have

$$
\mathrm{E}\left|X_{1}\right| I\left(\left|X_{1}\right|>t_{n}\right)=o\left(L\left(t_{n}\right) / t_{n}\right) \quad \text { as } n \rightarrow \infty,
$$

whenever $t_{n} \rightarrow \infty$ as $n \rightarrow \infty$. Hence

$$
\begin{aligned}
\mathrm{E} I_{2} & \leq \frac{2 \ell(n-\ell+1)}{\sqrt{\ell(n-\ell+1) L\left(z_{n}\right)}} \mathrm{E}\left|X_{1}\right| I\left(\left|X_{1}\right|>z_{n}\right) \\
& \leq \frac{2 \sqrt{\ell(n-\ell+1) L\left(z_{n}\right)}}{z_{n}} \frac{\mathrm{E}\left|X_{1}\right| I\left(\left|X_{1}\right|>z_{n}\right)}{L\left(z_{n}\right) / z_{n}} \\
& =o(1), \quad n \rightarrow \infty .
\end{aligned}
$$

The Lemma 2.5 follows now from (2.1), (2.2) and (2.4).

Consider the following inequality in order to prove Theorem 1.1.

$$
\begin{aligned}
& \sup _{1 / \ell \leq t \leq 1}\left|\frac{S_{[\ell t]}}{\sqrt{\ell} B_{n}}-\frac{W\left(s_{[\ell t]}^{2}\right)}{s_{\ell}}\right| \\
\leq & \max _{k \leq \ell}\left|\frac{S_{k}}{\sqrt{\ell} B_{n}}-\frac{S_{k}^{(n)}-\mathrm{E} S_{k}^{(n)}}{\sqrt{\ell} B_{n}}\right|+\max _{k \leq \ell}\left|\frac{S_{k}^{(n)}-\mathrm{E} S_{k}^{(n)}}{\nu_{\ell}}-\frac{S_{k}^{(n)}-\mathrm{E} S_{k}^{(n)}}{\sqrt{\ell} B_{n}}\right| \\
& +\max _{k \leq \ell}\left|\frac{S_{k}^{(n)}-\mathrm{E} S_{k}^{(n)}}{\nu_{\ell}}-\frac{W\left(s_{k}^{2}\right)}{\nu_{\ell}}\right|+\max _{k \leq \ell}\left|\frac{W\left(s_{k}^{2}\right)}{\nu_{\ell}}-\frac{W\left(s_{k}^{2}\right)}{s_{\ell}}\right| \\
= & J_{1}(n)+J_{2}(n)+J_{3}(n)+J_{4}(n) .
\end{aligned}
$$

Now we shall proceed the proof of Theorem 1.1 by dividing it into Propositions 2.1-2.4 below.

Proposition 2.1. Under the assumptions of Lemma 2.5, we have $J_{1}(n) \stackrel{\mathrm{P}}{\rightarrow} 0$ as $n \rightarrow \infty$.

Proof. By the Markov inequality and (2.3), we get

$$
\begin{aligned}
& \mathrm{P}\left\{\frac{1}{\sqrt{\ell L\left(z_{n}\right)}} \sum_{j=1}^{\ell}\left(\left|X_{j}\right| I\left\{\left|X_{j}\right|>z_{n}\right\}+\mathrm{E}\left|X_{j}\right| I\left\{\left|X_{j}\right|>z_{n}\right\}\right) \geq \varepsilon\right\} \\
\leq & \frac{2}{\varepsilon \sqrt{\ell L\left(z_{n}\right)}} \sum_{j=1}^{\ell} \mathrm{E}\left|X_{j}\right| I\left\{\left|X_{j}\right|>z_{n}\right\} \\
\leq & \frac{2}{\varepsilon \sqrt{\ell L\left(z_{n}\right)}} \ell \mathrm{E}\left|X_{1}\right| I\left\{\left|X_{1}\right|>z_{n}\right\} \\
= & \frac{2}{\varepsilon} \frac{\sqrt{\ell L\left(z_{n}\right)}}{z_{n}} \frac{\mathrm{E}\left|X_{1}\right| I\left\{\left|X_{1}\right|>z_{n}\right\}}{L\left(z_{n}\right) / z_{n}} \\
= & o(1)
\end{aligned}
$$


for any $\varepsilon>0$. It is well-known that there exist positive constants $C$ and $D$ such that

$$
C \leq \frac{\operatorname{Var}\left(S_{m}^{(n)}\right)}{m L\left(z_{n}\right)} \leq D
$$

for all $m=1,2, \ldots$ and $n$ large (see (3.10) in [4]). Hence it follows from Lemma 2.5 that

$$
\begin{aligned}
J_{1}(n) & \leq \frac{\nu_{\ell}}{\sqrt{\ell} B_{n}} \frac{\sqrt{\ell L\left(z_{n}\right)}}{\nu_{\ell}} \frac{1}{\sqrt{\ell L\left(z_{n}\right)}} \sum_{j=1}^{\ell}\left(\left|X_{j}\right| I\left\{\left|X_{j}\right|>z_{n}\right\}+\mathrm{E}\left|X_{j}\right| I\left\{\left|X_{j}\right|>z_{n}\right\}\right) \\
& \stackrel{\mathrm{P}}{\rightarrow} 0 \text { as } n \rightarrow \infty
\end{aligned}
$$

which completes the proof.

We next compute $J_{3}(n) \rightarrow 0$ as $n \rightarrow \infty$ in probability. It suffices from (2.6) to show that, as $n \rightarrow \infty$,

$$
\frac{1}{\sqrt{\ell L\left(z_{n}\right)}} \max _{k \leq \ell}\left|S_{k}^{(n)}-\mathrm{E} S_{k}^{(n)}-W\left(s_{k}^{2}\right)\right| \stackrel{\mathrm{P}}{\rightarrow} 0 .
$$

To prove this we shall use a blocking argument. Define blocks of integers $H_{1}, I_{1}, H_{2}, I_{2}, \ldots$ by requiring that $H_{k}$ contains $h_{k}$ and $I_{k}$ contains $i_{k}$ consecutive integers and that there are no gaps between consecutive blocks, where

$$
\begin{aligned}
& h_{k}=\operatorname{Card} H_{k}=\left[a k^{a-1} \exp \left(k^{a}\right)\right], \\
& i_{k}=\operatorname{Card} I_{k}=\left[a k^{a-1} \exp \left(k^{a} / 2\right)\right]
\end{aligned}
$$

for some $0<a<1$. Put

$$
\begin{aligned}
& N_{k}=\sum_{j \leq k} \operatorname{Card}\left(H_{j} \cup I_{j}\right) \sim \exp \left(k^{a}\right), \\
& u_{k}=\sum_{j \in H_{k}}\left(Y_{j, n}-\mathrm{E} Y_{j, n}\right), \quad v_{k}=\sum_{j \in I_{k}}\left(Y_{j, n}-\mathrm{E} Y_{j, n}\right) .
\end{aligned}
$$

Clearly, for each $n$ there exists a unique $m_{n}$ such that $N_{m_{n}} \leq n<N_{m_{n}+1}$. Hence $m_{n} \sim(\log n)^{1 / a}$ and $N_{m_{n}} \sim n$. Let

$$
\tilde{\sigma}_{i}^{2}=\mathrm{E} u_{i}^{2}, \quad \tilde{s}_{m}^{2}=\sum_{i=1}^{m} \tilde{\sigma}_{i}^{2}, \quad s_{n}^{2}=\tilde{s}_{m_{n}}^{2} .
$$

Then we see that

$$
S_{k}^{(n)}-\mathrm{E} S_{k}^{(n)}=\sum_{i=1}^{m_{k}} u_{i}+\sum_{i=1}^{m_{k}} v_{i}+\sum_{j=N_{m_{k}}+1}^{k}\left(Y_{j, n}-\mathrm{E} Y_{j, n}\right) .
$$

The following lemma corresponds to Lemma 9.2.4 in [9]. 
Lemma 2.6. Let $u_{k}, v_{k}$ be as above. Suppose that $\sum_{n=1}^{\infty} \rho\left(2^{n}\right)<\infty$. Then there exists a constant $C=C(\rho(\cdot))$ such that for any $k \geq 0, n \geq 1$

$$
\mathrm{E}\left(\sum_{i=k+1}^{k+n} u_{i}\right)^{2} \leq C \sum_{i=k+1}^{k+n} \mathrm{E} u_{i}^{2}, \quad \mathrm{E}\left(\sum_{i=k+1}^{k+n} v_{i}\right)^{2} \leq C \sum_{i=k+1}^{k+n} \mathrm{E} v_{i}^{2} .
$$

Lemma 2.7 ([1]). If

$$
\sum_{k=1}^{\infty} \phi^{1 / 2}\left(e^{k^{a} / 2}\right)<\infty
$$

then without changing its distribution, we can redefine the sequence $\left\{u_{i}\right\}_{i \geq 1}$ on a larger probability space together with a sequence $\left\{\bar{Y}_{i}\right\}_{i \geq 1}$ of independent random variables such that

$$
\begin{aligned}
& \bar{Y}_{k} \stackrel{\mathcal{D}}{=} u_{k} \quad \text { for all } k, \\
& \left|\sum_{k=1}^{m} u_{k}-\sum_{k=1}^{m} \bar{Y}_{k}\right| \leq C \quad \text { a.s. for all } m \text { and some constant } C .
\end{aligned}
$$

The next lemma is a well-known Sakhanenko's theorem (cf. Lemma 2 in $[6])$.

Lemma 2.8. Let $X_{1}, X_{2}, \ldots$ be independent random variables with $\mathrm{E} X_{j}=0$ and $\sigma_{j}^{2}=\mathrm{E} X_{j}^{2}<\infty$ for each $j \geq 1$. Then we can redefine $\left\{X_{j}, j \geq 1\right\}$ on a richer probability space together with a sequence of independent $N(0,1)$-random variables $\tilde{Y}_{j}, j \geq 1$, such that for every $p>2$ and $x>0$,

$$
\mathrm{P}\left\{\max _{i \leq n}\left|\sum_{j=1}^{i} X_{i}-\sum_{j=1}^{i} \sigma_{j} \tilde{Y}_{i}\right| \geq x\right\} \leq(A p)^{p} x^{-p} \sum_{j=1}^{n} \mathrm{E}\left|X_{j}\right|^{p},
$$

where $A$ is an absolute positive constant.

In view of Lemma 2.8, without changing its distribution we can redefine the sequence $\left\{\bar{Y}_{i}\right\}_{i \geq 1}$ together with a sequence $\left\{\tilde{Y}_{i}\right\}_{i \geq 1}$ of independent normal random variables with $\mathrm{E} \tilde{Y}_{i}=0, \mathrm{E} \tilde{Y}_{i}^{2}=\tilde{\sigma}_{i}^{2}$ such that, for some $\delta>0$,

$$
\mathrm{P}\left\{\max _{m \leq M}\left|\sum_{i=1}^{m} \bar{Y}_{i}-\sum_{i=1}^{m} \tilde{Y}_{i}\right| \geq x\right\} \leq \frac{C}{x^{2+\delta}} \sum_{i=1}^{M} \mathrm{E}\left|\bar{Y}_{i}\right|^{2+\delta} .
$$

Furthermore, without changing its distribution we can redefine the sequence $\left\{\tilde{Y}_{i}\right\}_{i \geq 1}$ together with a standard Wiener process $W=\{W(t)\}_{t \geq 0}$ such that

$$
W\left(\tilde{s}_{m}^{2}\right)=\sum_{i=1}^{m} \tilde{Y}_{i} \quad \text { for every } m
$$


By (2.8), we have, for any $\varepsilon>0$,

$$
\begin{aligned}
& \mathrm{P}\left\{\max _{k \leq \ell}\left|S_{k}^{(n)}-\mathrm{E} S_{k}^{(n)}-W\left(s_{k}^{2}\right)\right|>\varepsilon \sqrt{\ell L\left(z_{n}\right)}\right\} \\
\leq & \mathrm{P}\left\{\max _{m \leq m_{\ell}}\left|\sum_{i=1}^{m} u_{i}-\sum_{i=1}^{m} \bar{Y}_{i}\right|>\frac{\varepsilon}{4} \sqrt{\ell L\left(z_{n}\right)}\right\} \\
& +\mathrm{P}\left\{\max _{m \leq m_{\ell}}\left|\sum_{i=1}^{m} v_{i}\right|>\frac{\varepsilon}{4} \sqrt{\ell L\left(z_{n}\right)}\right\} \\
& +\mathrm{P}\left\{\max _{m \leq m_{\ell}} N_{m} \leq k<N_{m+1}\left|\sum_{j=N_{m}+1}^{k}\left(Y_{j, n}-\mathrm{E} Y_{j, n}\right)\right|>\frac{\varepsilon}{4} \sqrt{\ell L\left(z_{n}\right)}\right\} \\
& +\mathrm{P}\left\{\max _{m \leq m_{\ell}}\left|\sum_{i=1}^{m} \bar{Y}_{i}-\sum_{i=1}^{m} \tilde{Y}_{i}\right|>\frac{\varepsilon}{4} \sqrt{\ell L\left(z_{n}\right)}\right\} \\
=: & P_{1}(n)+P_{2}(n)+P_{3}(n)+P_{4}(n) .
\end{aligned}
$$

From Lemma 2.7, it is immediate that

$$
P_{1}(n)=0 \text { for } n \text { large. }
$$

Lemma 2.9. Under the assumptions of Lemma 2.5, we have $P_{2}(n) \rightarrow 0$ as $n \rightarrow \infty$.

Proof. It follows that, for some $0<a<1$,

$$
\sum_{k=1}^{m_{\ell}} i_{k}=\sum_{k=1}^{m_{\ell}}\left[a k^{a-1} \exp \left(k^{a} / 2\right)\right] \leq C \sqrt{\ell} .
$$

Hence, according to Lemmas 2.1 and 2.6, we have, for every $m \leq m_{\ell}$,

$$
\begin{aligned}
\mathrm{E}\left(\sum_{k=1}^{m} v_{k}\right)^{2} & \leq C \sum_{k=1}^{m} \mathrm{E} v_{k}^{2} \leq C \sum_{k=1}^{m} i_{k} \mathrm{E}\left\{Y_{j, n}-\mathrm{E} Y_{j, n}\right\}^{2} \\
& \leq C L\left(z_{n}\right) \sum_{k=1}^{m} i_{k} \leq C \sqrt{\ell} L\left(z_{n}\right) .
\end{aligned}
$$

Using Lemma 2.2 in [11], we get

$$
\mathrm{E} \max _{m \leq m_{\ell}}\left(\sum_{k=1}^{m} v_{k}\right)^{2} \leq C \sqrt{\ell} L\left(z_{n}\right) .
$$

The result follows by the Chebyshev's inequality.

Lemma 2.10. Under the assumptions of Lemma 2.5, we have $P_{3}(n) \rightarrow 0$ as $n \rightarrow \infty$. 
SELF-NORMALIZED WEAK LIMIT THEOREMS FOR A $\phi$-MIXING SEQUENCE 1149

Proof. By the Markov's inequality, for every $\delta>0$,

$$
\begin{aligned}
P_{3}(n) & \leq \sum_{m=1}^{m_{\ell}} \mathrm{P}\left\{\max _{N_{m} \leq k<N_{m+1}}\left|\sum_{j=N_{m}+1}^{k}\left(Y_{j, n}-\mathrm{E} Y_{j, n}\right)\right|>\frac{\varepsilon}{4} \sqrt{\ell L\left(z_{n}\right)}\right\} \\
& \leq \frac{C}{\left(\ell L\left(z_{n}\right)\right)^{(2+\delta) / 2}} \sum_{m=1}^{m_{\ell}} \mathrm{E}\left\{\max _{N_{m} \leq k<N_{m+1}}\left|\sum_{j=N_{m}+1}^{k}\left(Y_{j, n}-\mathrm{E} Y_{j, n}\right)\right|\right\}^{2+\delta} .
\end{aligned}
$$

Applying Lemma 2.3 for $\delta>\delta_{0}$, we obtain

$$
\begin{aligned}
& \mathrm{E}\left\{\max _{N_{m} \leq k<N_{m+1}}\left|\sum_{j=N_{m}+1}^{k}\left(Y_{j, n}-\mathrm{E} Y_{j, n}\right)\right|\right\}^{2+\delta} \\
\leq & K\left\{\operatorname{Var}\left(\sum_{j=N_{m}+1}^{N_{m+1}}\left(Y_{j, n}-\mathrm{E} Y_{j, n}\right)\right)\right\}^{(2+\delta) / 2} \\
\leq & K\left\{\mathrm{E}\left(\sum_{j=N_{m}+1}^{N_{m+1}}\left(Y_{j, n}-\mathrm{E} Y_{j, n}\right)\right)^{2}\right\}^{(2+\delta) / 2},
\end{aligned}
$$

and, by Lemma 2.1,

$$
\begin{aligned}
\mathrm{E}\left(\sum_{j=N_{m}+1}^{N_{m+1}}\left(Y_{j, n}-\mathrm{E} Y_{j, n}\right)\right)^{2} & \leq C\left(N_{m+1}-N_{m}\right) \mathrm{E}\left(Y_{j, n}-\mathrm{E} Y_{j, n}\right)^{2} \\
& \leq C h_{m} L\left(z_{n}\right) .
\end{aligned}
$$

It is easily seen that

$$
\sum_{m=1}^{m_{\ell}} h_{m}^{(2+\delta) / 2}=\sum_{m=1}^{m_{\ell}} o\left(m^{a-1} e^{(2+\delta) m^{a} / 2}\right)=o\left(e^{(2+\delta) m_{\ell}^{a} / 2}\right)=o\left(\ell^{(2+\delta) / 2}\right)
$$

for some $0<a<1$. Combining the above results, we have

$$
P_{3}(n) \leq \frac{C}{\left(\ell L\left(z_{n}\right)\right)^{(2+\delta) / 2}} \sum_{m=1}^{m_{\ell}} h_{m}^{(2+\delta) / 2}\left\{L\left(z_{n}\right)\right\}^{(2+\delta) / 2}=o(1) .
$$

This completes the proof of Lemma 2.10.

Lemma 2.11. Under the assumptions of Lemma 2.5, we have $P_{4}(n) \rightarrow 0$ as $n \rightarrow \infty$.

Proof. Using Lemmas 2.1, 2.3 and 2.7, we have, for $\delta>\delta^{\prime}>0$

$$
\begin{aligned}
\mathrm{E}\left|\bar{Y}_{i}\right|^{2+\delta} & =\mathrm{E}\left|u_{i}\right|^{2+\delta} \leq K\left(\operatorname{Var}\left(\sum_{j \in H_{i}}\left(Y_{j, n}-\mathrm{E} Y_{j, n}\right)\right)\right)^{(2+\delta) / 2} \\
& =K\left(\mathrm{E}\left\{\sum_{j \in H_{i}}\left(Y_{j, n}-\mathrm{E} Y_{j, n}\right)\right\}^{2}\right)^{(2+\delta) / 2}
\end{aligned}
$$




$$
\leq C h_{i}^{(2+\delta) / 2}\left(L\left(z_{n}\right)\right)^{(2+\delta) / 2} \text {. }
$$

Hence, together with (2.14), we have

$$
\begin{aligned}
\frac{1}{\left\{\ell L\left(z_{n}\right)\right\}^{1+\delta / 2}} \sum_{i=1}^{m_{\ell}} \mathrm{E}\left|\bar{Y}_{i}\right|^{2+\delta} & \leq \frac{C}{\left(\ell L\left(z_{n}\right)\right)^{1+\delta / 2}} \sum_{i=1}^{m_{\ell}} h_{i}^{(2+\delta) / 2}\left(L\left(z_{n}\right)\right)^{(2+\delta) / 2} \\
& \rightarrow 0 \quad \text { as } n \rightarrow \infty .
\end{aligned}
$$

By (2.9), we obtain

$$
P_{4}(n) \rightarrow 0 \text { as } n \rightarrow \infty
$$

Combining (2.11), Lemmas 2.9-2.11 and (2.10), we have the following result.

Proposition 2.2. If $\phi(1)<1$ and $\sum_{n=1}^{\infty} \phi^{1 / 2}(n)<\infty$, then $J_{3}(n) \stackrel{\mathrm{P}}{\rightarrow} 0$ as $n \rightarrow \infty$.

Proposition 2.3. Under the assumptions of Lemma 2.5, we have $J_{4}(n) \stackrel{\mathrm{P}}{\rightarrow} 0$ as $n \rightarrow \infty$.

Proof. We have

$$
J_{4}(n)=\left|\frac{s_{\ell}}{\nu_{\ell}}-1\right| \max _{k \leq \ell} \frac{\left|W\left(s_{k}^{2}\right)\right|}{s_{\ell}} .
$$

It is easy to check that

$$
\max _{k \leq \ell} \frac{\left|W\left(s_{k}^{2}\right)\right|}{s_{\ell}} \stackrel{\mathrm{P}}{\rightarrow} C \quad \text { as } \ell \rightarrow \infty
$$

for some positive constant $C$. Thus we only need to show that $s_{\ell} \sim \nu_{\ell}$. Since

$$
\frac{s_{\ell}^{2}}{\nu_{\ell}^{2}}-1=\frac{1}{\gamma_{\ell}^{2}} \frac{1}{\ell L\left(z_{n}\right)}\left(s_{\ell}^{2}-\operatorname{Var}\left(S_{\ell}^{(n)}\right)\right),
$$

it is sufficient to show that

$$
\frac{1}{\ell L\left(z_{n}\right)}\left(s_{\ell}^{2}-\operatorname{Var}\left(S_{\ell}^{(n)}\right)\right) \rightarrow 0 \quad \text { as } n \rightarrow \infty .
$$

From (2.8) we have

$$
\begin{aligned}
\operatorname{Var}\left(S_{\ell}^{(n)}\right)= & \mathrm{E}\left(\sum_{k=1}^{m_{\ell}} u_{k}\right)^{2}+\mathrm{E}\left(\sum_{k=1}^{m_{\ell}} v_{k}+\sum_{j=N_{m_{\ell}}+1}^{\ell}\left(Y_{j, n}-\mathrm{E} Y_{j, n}\right)\right)^{2} \\
& +2 \mathrm{E}\left\{\left(\sum_{k=1}^{m_{\ell}} u_{k}\right)\left(\sum_{k=1}^{m_{\ell}} v_{k}+\sum_{j=N_{m_{\ell}}+1}^{\ell}\left(Y_{j, n}-\mathrm{E} Y_{j, n}\right)\right)\right\} .
\end{aligned}
$$


SELF-NORMALIZED WEAK LIMIT THEOREMS FOR A $\phi$-MIXING SEQUENCE 1151

Noting that $\sum_{i=1}^{m_{\ell}} h_{i} \leq C \ell$, it follows from Kronecker's lemma and Toeplitz's lemma that

$$
\begin{aligned}
\mathrm{E}\left(\sum_{k=1}^{m_{\ell}} u_{k}\right)^{2}-s_{\ell}^{2} & =2 \sum_{k=1}^{m_{\ell}-1} \sum_{j=k+1}^{m_{\ell}} \mathrm{E} u_{k} u_{j} \\
& \leq C \sum_{k=1}^{m_{\ell}-1} \sum_{j=k+1}^{m_{\ell}} \phi^{1 / 2}\left(i_{k}\right)\left(\mathrm{E} u_{k}^{2}\right)^{1 / 2}\left(\mathrm{E} u_{j}^{2}\right)^{1 / 2} \\
& \leq C \sum_{j=2}^{m_{\ell}} h_{j}^{1 / 2} \sum_{k=1}^{j-1} \phi^{1 / 2}\left(i_{k}\right) h_{k}^{1 / 2} L\left(z_{n}\right) \\
& =C \sum_{j=2}^{m_{\ell}} h_{j}\left(o\left(h_{j}^{1 / 2}\right) / h_{j}^{1 / 2}\right) L\left(z_{n}\right) \\
& =o\left(\sum_{j=2}^{m_{\ell}} h_{j}\right) L\left(z_{n}\right)=o\left(\ell L\left(z_{n}\right)\right) .
\end{aligned}
$$

Using (2.13), Lemma 2.1 and the property of $N_{k}$, we have

$$
\begin{aligned}
& \mathrm{E}\left(\sum_{k=1}^{m_{\ell}} v_{k}+\sum_{j=N_{m_{\ell}}+1}^{\ell}\left(Y_{j, n}-\mathrm{E} Y_{j, n}\right)\right)^{2} \\
\leq & 2\left\{\mathrm{E}\left(\sum_{k=1}^{m_{\ell}} v_{k}\right)^{2}+\mathrm{E}\left(\sum_{j=N_{m_{\ell}}+1}^{\ell}\left(Y_{j, n}-\mathrm{E} Y_{j, n}\right)\right)^{2}\right\} \\
\leq & C\left(\sqrt{\ell} L\left(z_{n}\right)+\left(\ell-N_{m_{\ell}}\right) L\left(z_{n}\right)\right) \\
= & o\left(\ell L\left(z_{n}\right)\right) .
\end{aligned}
$$

On the other hand, the Hölder's inequality yields

$$
\begin{aligned}
& \mathrm{E}\left|\left(\sum_{k=1}^{m_{\ell}} u_{k}\right)\left(\sum_{k=1}^{m_{\ell}} v_{k}+\sum_{j=N_{m_{\ell}}+1}^{\ell}\left(Y_{j, n}-\mathrm{E} Y_{j, n}\right)\right)\right| \\
\leq & \left\{\mathrm{E}\left(\sum_{k=1}^{m_{\ell}} u_{k}\right)^{2}\right\}^{1 / 2}\left\{\mathrm{E}\left(\sum_{k=1}^{m_{\ell}} v_{k}+\sum_{j=N_{m_{\ell}+1}}^{\ell}\left(Y_{j, n}-\mathrm{E} Y_{j, n}\right)\right)^{2}\right\}^{1 / 2} \\
\leq & C\left\{\ell L\left(z_{n}\right)\right\}^{1 / 2}\left\{\left(\sqrt{\ell}+\ell-N_{m_{\ell}}\right) L\left(z_{n}\right)\right\}^{1 / 2} \\
= & o\left(\ell L\left(z_{n}\right)\right) .
\end{aligned}
$$

Substituting (2.17)-(2.19) into (2.16) yields (2.15). This completes the proof.

Proposition 2.4. Under the assumptions of Proposition 2.2, we have $J_{2}(n) \stackrel{\mathrm{P}}{\rightarrow}$ 0 as $n \rightarrow \infty$. 
Proof. Note that

$$
\begin{aligned}
J_{2}(n) & =\left|\frac{1}{\nu_{\ell}}-\frac{1}{\sqrt{\ell} B_{n}}\right| \max _{k \leq \ell}\left|S_{k}^{(n)}-\mathrm{E} S_{k}^{(n)}\right| \\
& =\left|1-\frac{\nu_{\ell}}{\sqrt{\ell} B_{n}}\right| \max _{k \leq \ell} \frac{\left|S_{k}^{(n)}-\mathrm{E} S_{k}^{(n)}\right|}{\nu_{\ell}} \\
& \leq\left|1-\frac{\nu_{\ell}}{\sqrt{\ell} B_{n}}\right|\left(J_{3}(n)+J_{4}(n)+\max _{k \leq \ell} \frac{\left|W\left(s_{k}^{2}\right)\right|}{s_{\ell}}\right) .
\end{aligned}
$$

Combining Lemma 2.5 with Propositions $2.2-2.3$ gives $J_{2}(n) \stackrel{\mathrm{P}}{\rightarrow} 0$ as $n \rightarrow$ $\infty$.

Proof of Theorem 1.1. The proof is immediate from (2.5) and Propositions 2.12.4 .

Proof of Theorem 1.2. The first statement of Theorem 1.2 is immediate from the second one. Hence we need only to prove the second one. By the Slutsky lemma, we see that $W\left(s_{[\ell t]}^{2}\right) / s_{\ell} \stackrel{\mathcal{D}}{=} W\left(s_{[\ell t]}^{2} / s_{\ell}^{2}\right)$ for all $0 \leq t \leq 1$. Thus, it suffices to show that

We consider

$$
\sup _{0 \leq t \leq 1}\left|W\left(\frac{s_{[\ell t]}^{2}}{s_{\ell}^{2}}\right)-W(t)\right| \stackrel{\mathrm{P}}{\rightarrow} 0 \quad \text { as } \ell \rightarrow \infty .
$$

$$
\begin{aligned}
& \sup _{0 \leq t \leq 1}\left|W\left(\frac{s_{[\ell t]}^{2}}{s_{\ell}^{2}}\right)-W(t)\right| \\
\leq & \sup _{0 \leq t \leq 1}\left|W\left(\frac{s_{[\ell t]}^{2}}{s_{\ell}^{2}}\right)-W\left(\frac{[\ell t]}{\ell}\right)\right|+\sup _{0 \leq t \leq 1}\left|W\left(\frac{[\ell t]}{\ell}\right)-W(t)\right| \\
= & : Q_{1}(n)+Q_{2}(n) .
\end{aligned}
$$

By the uniform continuity of Wiener process, $Q_{2}(n)=o(1)$ a.s. If now we prove that

$$
\sup _{0 \leq t \leq 1}\left|\frac{s_{[\ell t]}^{2}}{s_{\ell}^{2}}-\frac{[\ell t]}{\ell}\right|=o(1),
$$

then this, together with the Etemadi's maximal inequality (cf. [7]) and the Markov's inequality, gives $Q_{1}(n) \stackrel{\mathrm{P}}{\rightarrow} 0$. Indeed, by Lemma 3.5 in [14], $\nu_{\ell}^{2}$ is regularly varying at $\infty$ with exponent 1 . Since $s_{\ell}^{2} \sim \nu_{\ell}^{2}$ in the proof of Proposition 2.3, we can obtain (2.20) by the uniform convergence theorem of a regularly varying function (see Theorem 1.5.2 in [3]). This completes the proof of Theorem 1.2. 
Acknowledgement. The authors wish to thank the referee for careful reading and helpful comments on this paper.

\section{References}

[1] R. Balan and R. Kulik, Weak invariance principle for mixing sequences in the domain of attraction of normal law, Studia Sci. Math. Hungarica 46 (2009), no. 3, 329-343.

[2] R. Balan and I. M. Zamfirescu, Strong approximation for mixing sequences with infinite variance, Electron. Comm. Probab. 11 (2006), 11-23.

[3] N. H. Bingham, C. M. Goldie, and J. L. Teugels, Regular Variation, Cambridge University Press, Cambridge, 1987.

[4] R. C. Bradley, A central limit theorem for stationary $\rho$-mixing sequences with infinite variance, Ann. Probab. 16 (1988), no. 1, 313-332.

[5] M. Csörgö, Z. Y. Lin, and Q. M. Shao, Studentized increments of partial sums, Sci. China Ser. A. 37 (1994), no. 3, 265-276.

[6] M. Csörgő, B. Szyszkowicz, and Q. Wang, Donsker's theorem for self-normalized partial sums processes, Ann. Probab. 31 (2003), no. 3, 1228-1240.

[7] N. Etemadi, On some classical results in probability theory, Sankhya Ser. A 47 (1985), no. 2, 215-221.

[8] P. Griffin and J. Kuelbs, Some extensions of the LIL via self-normalizations, Ann. Probab. 19 (1991), no. 1, 380-395.

[9] Z. Y. Lin and C. R. Lu, Limit Theory for Mixing Dependent Random Variables, Kluwer Academic Publishers, Dordrecht; Science Press, New York, 1996.

[10] M. Peligrad, The convergence of moments in the central limit theorem for $\rho$-mixing sequences of random variables, Proc. Amer. Math. Soc. 101 (1987), no. 1, 142-148.

[11] M. Peligrad and Q. M. Shao, Estimation of the variance of partial sums for $\rho$-mixing random variables, J. Multivariate Anal. 52 (1995), no. 1, 140-157.

[12] A. Račkauskas and C. Suquet, Invariance principles for adaptive self-normalized partial sums processes, Stochastic Process. Appl. 95 (2001), no. 1, 63-81.

[13] Q. M. Shao, Almost sure invariance principles for mixing sequences of random variables, Stochastic Process. Appl. 48 (1993), no. 2, 319-334.

[14] _ An invariance principle for stationary $\rho$-mixing sequence with infinite variance, Chinese Ann. Math. Ser. B 14 (1993), no. 1, 27-42.

[15] _ Self-normalized large deviations, Ann. Probab. 25 (1997), no. 1, 285-328.

[16] W. Wang, Self-normalized lag increments of partial sums, Statist. Probab. Lett. 58 (2002), no. 1, 41-51.

YonG-KAB CHOI

Department of Mathematics and RINS

Gyeongsang National University

JiNJU 660-701, KOREA

E-mail address: mathykc@naver.com

HeE-Jin Moon

Department of Mathematics and RINS

Gyeongsang National University

JiNJU 660-701, KoREA

E-mail address: mhj0307@daum.net 